\title{
AN ATTACHMENT FOR TURNING APPROXIMATELY SPHERICAL SURFACES OF SMALL CURVATURE ON A LATHE
}

\author{
By I. C. Gardner
}

ABSTRACT

This device replaces the compound rest on a lathe and was particularly designed for the production of the convex or concave surfaces of lens-grinding tools although it is well adapted for the production of such surfaces for any purpose. It is intended to be used only for the production of surfaces of which the radius of curvature is too great to permit the use of a tool mounted on the end of a radius rod. The mathematical theory underlying the design and a detailed description of the instrument as built are given in detail. The attachment, as constructed, permits disks under $300 \mathrm{~mm}$ in diameter to be faced and any radius of curvature, greater than $500 \mathrm{~mm}$ may be obtained.

The surfaces produced are not accurately spherical, but for many purposes the approximation is entirely satisfactory. The approximation becomes poorer as either the curvature or diameter of the surface is increased. For a surface 200 $\mathrm{mm}$ in diameter with a radius of curvature of $1,000 \mathrm{~mm}$ the maximum departure from sphericity is $0.02 \mathrm{~mm}$. If the diameter of the tool is $300 \mathrm{~mm}$ and the radius of curvature is $500 \mathrm{~mm}$, the departure from sphericity is approximately $0.3 \mathrm{~mm}$. This last value is the maximum departure for any surface lying within the working range of the instrument.

\section{CONTENTS}

Page

I. Introduction

II. General description of the attachment 228

III. Derivation of the equation of the curve generated by the linkage

IV. Dimensions and performance characteristics of linkage adopted for

$\begin{array}{ll}\text { construction } & 232 \\ \text { V. Mechanical details of the completed instrument... }\end{array}$

\section{INTRODUCTION}

In the construction of machines or tools it is often necessary to form a small portion of a spherical surface of which the radius of curvature is very long. This is particularly the case when making lens-grinding tools. The tool employed for grinding or polishing the surface of a lens may be described as a circular disk, usually of brass or cast iron, with a hub at the center of one face by which it can be mounted on a spindle and with the other face, in special cases plane, but generally convex or concave, the radius of curvature being the same in magnitude but opposite in sense to that of the surface desired on the finished lens.

When the radius of curvature is not too great such surfaces can be conveniently generated by mounting the cutting tool of a lathe on the end of a radius rod of the desired length. However, if the radius of curvature is much greater than $2,000 \mathrm{~mm}$ such a method is not 
suitable, and convenient methods for producing such surfaces are not commonly available in the machine shop. There is a machine tool by which spherical surfaces of small curvature can be generated but its method of operation requires the use of a milling cutter with its diameter at least as great as that of the lens tool to be made. It follows that a machine of this type must be large and strongly built It is necessarily expensive and can not be used economically unless the demand for spherical surfaces is such that it can be kept in operation for long periods of time.

There are other devices on the market for producing tools for the manufacture of ophthalmic lenses. Some of these are not fitted for making a surface with the radius of curvature greater than 2 or $3 \mathrm{~m}$. Others, which permit surfaces of less curvature to be made, employ templates, and hence do not permit any desired curvature to be obtained except by the construction of a special template.

Accordingly, when the radius of curvature is large, the difficulty of readily producing such a surface, by methods hitherto available, is so great that, in some instances, the lens designer actually avoids the use of surfaces of small curvature although this course is not satisfactory except for certain types of optical systems. In other cases, arcs are described on sheet metal by a marker on the end of the required length of piano wire. Two templates are filed from the metal-the one convex, the other concave-and these are ground together to eliminate the smaller irregularities. These templates are used to control the form of the tool which is turned by hand on a lathe. Hand turning would not be sufficiently precise except that lens tools are usually required in pairs, convex and concave, and the two tools can be ground together before use. The turning of tools by such a method is not only costly and tedious, but the cost is often further increased by the excessive amount of grinding which is required to prepare hand-turned tools for use.

Because of these difficulties a new method of producing approximately spherical surfaces of small curvature has been developed. By this method the surfaces may be produced on a lathe by substituting a relatively simple attachment for the compound rest. An adjustment can be readily made to give any radius of curvature within the range of the attachment. The sharper curvatures can not be generated by this device, but this offers no serious disadvantages as simple and satisfactory methods are already available for such curves. The surfaces generated are not truly spherical but in many cases, particularly for the larger radii of curvature, or for tools of small diameter, the departure from sphericity is so small that the tools may be used as turned. For the sharper curvatures falling within the range of the instrument it may be necessary to grind the members of a pair of tools together, but the time of grinding will be small as compared with that required for tools turned by hand.

\section{GENERAL DESCRIPTION OF THE ATTACHMENT}

According to this method the spherical surface to be produced is turned on a lathe by a single-tooth tool mounted in a special tool holder which is guided to generate the desired surface by a simple linkage system. A plan view of the fundamental parts is shown diagrammatically in Figure 1. The line $K N$ is the axis of the lathe 
spindle and the arc $K L$ is a meridian section of the curved surface which is turned by the cutting tool indicated at $K$. At $A$ and $B$ are two pins which are fixed to the lathe carriage. The triangle $C D E$, on which the cutting tool is rigidly mounted, is pushed across the lathe by a cross-feed and, as it advances, the two sides remain in contact with the pins $A$ and $B$, thereby causing the triangle to rotate
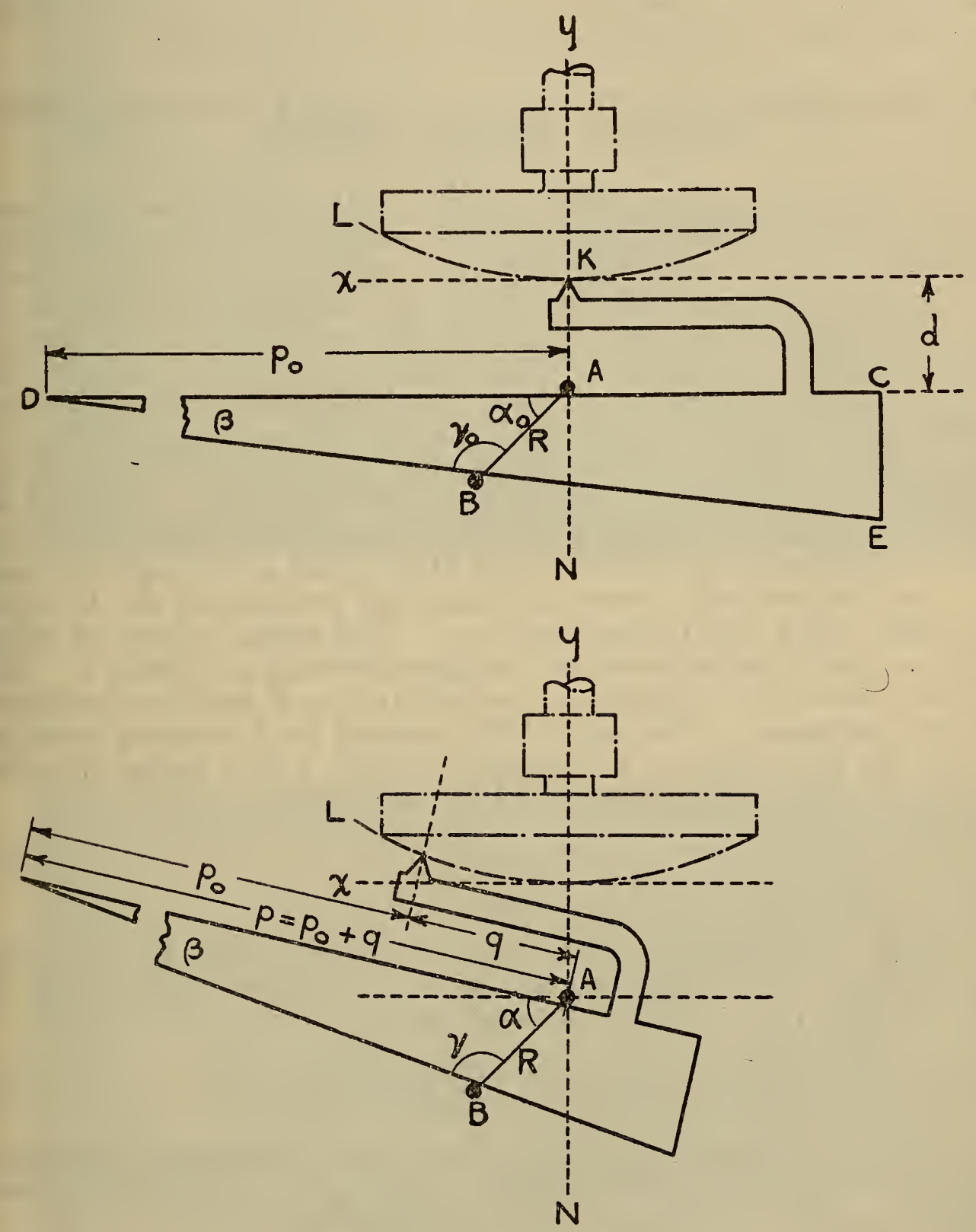

Figure 1.-Diagrammatic sketch of linkage

in such a manner that the cutting tool generates the arc $K L$. When the lathe spindle is revolving, therefore, a surface of revolution with the arc $K L$ as a meridian section is produced.

Under the guidance of this linkage, the tool generates an arc which is not truly circular, but the approximation is satisfactory over the entire range of curvatures for which the use of a radius bar is unsatisfactory. Therefore the two methods supplement each other and, together, provide for the production of any curvature. The amount 
of curvature is determined by the magnitude of the angle between lines $D E$ and $D C$ and by the length $A B$. It is convenient to hold $A B$ constant and provide means for varying the angle. The instrument may be calibrated to permit a setting to be readily made for any desired curvature. It is an advantage of this linkage that the constraints at $A$ and $B$ do not change their directions of action during the production of a given surface and accordingly inaccuracies, which may arise from lost motion, are substantially eliminated.

\section{DERIVATION OF THE EQUATION OF THE CURVE GEN- ERATED BY THE LINKAGE}

In order to determine the exact form of the surface generated by this attachment it is desirable to derive the equation of the meridian section, which is the plane curve generated by the linkage system. In Figure 1 the essential portions of the linkage are illustrated in two positions. In the upper drawing the triangle $C D E$ is shown in its initial position with $D C$ at right angles to the bed of the lathe and with $K$, the generating point, in the prolongation of the axis of the spindle of the lathe. As this triangle is advanced across the bed of the lathe the sides $D C$ and $D E$ remain in contact with the fixed constraints $A$ and $B$, thus causing the triangle in the course of its motion to assume the position shown in the lower drawing of Figure 1. Two pardmeters, $p$, the distance from $D$ to $A$, and $a$, the angle between the lines $A B$ and $C D$, serve to define the position of the triangle. The angle $D B A$ will be denoted by $\gamma$. The initial values of these lengths $A B$ and $\gamma_{0}$ as indicated in the upper drawing of Figure 1. The of the instrument. during the to produce different curvatures.

$$
p-p_{0}=q
$$

where $q$ is the extent of travel of $D C$ past $A$.

The $x y$ system of coordinates indicated on Figure 1 is assumed fixed with respect to the bed of the lathe and the parts $A$ and $B$.
Then, for any point on the generated curve,

$$
\begin{aligned}
& x=q \cos \left(a-a_{0}\right)-d \sin \left(a-a_{0}\right) \\
& y=q \sin \left(a-a_{0}\right)+d\left[\cos \left(a-a_{0}\right)-1\right]
\end{aligned}
$$

but for the more convenient application of these equations it is desirable that the trigonometric functions of $\left(a-a_{0}\right)$ be replaced by equiva-
lent expressions in terms of $\beta$. If, by definition

then

$$
\begin{aligned}
& P=\frac{p}{R} \sin \beta \\
& Q=\left(1-P^{2}\right)^{1 / 2}
\end{aligned}
$$

Since

$$
\begin{aligned}
& \sin \gamma=P \\
& \cos \gamma=-Q
\end{aligned}
$$

$$
a=180^{\circ}-(\beta+\gamma)
$$




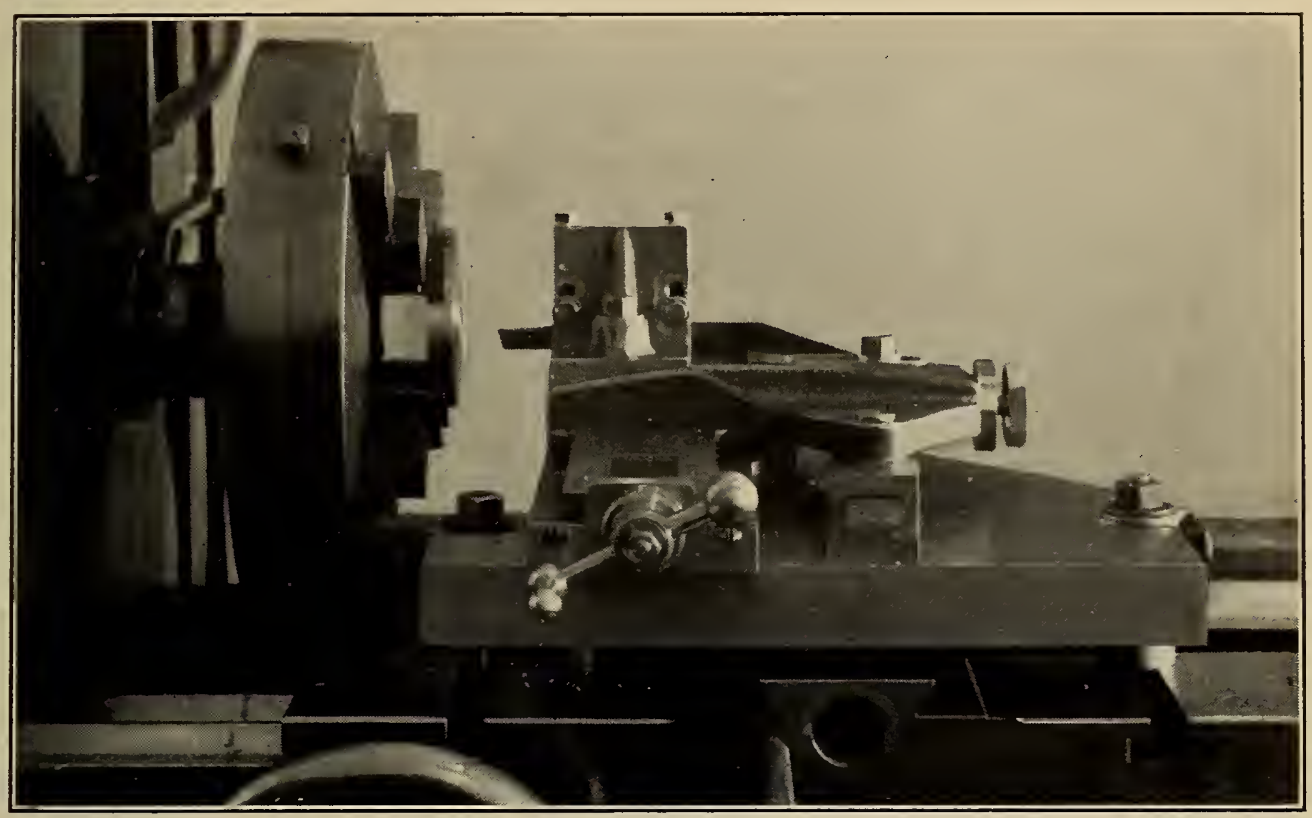

FIGURE 3.-Front view of attachment mounted on a lathe

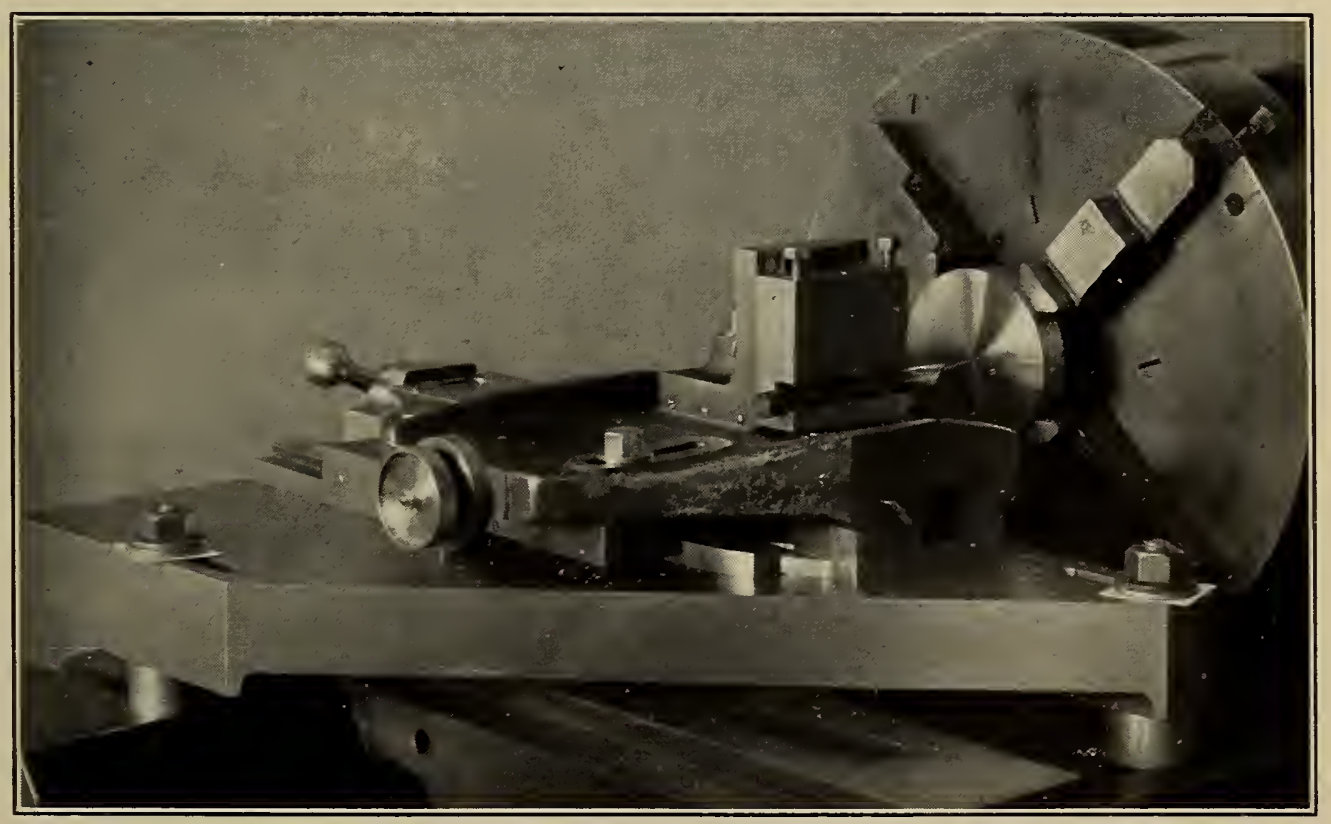

FIGURE 4.-Back view of attachment mounted on a lathe 


$$
\sin a=\sin (\beta+\gamma)=P \cos \beta-Q \sin \beta \text { and }
$$

Similarly

$$
\cos a=-\cos (\beta+\gamma)=Q \cos \beta+P \sin \beta \text {. }
$$

$$
\begin{aligned}
& \sin a_{0}=P_{0} \cos \beta-Q_{0} \sin \beta \\
& \cos a_{0}=Q_{0} \cos \beta+P_{0} \sin \beta
\end{aligned}
$$

where

$$
\begin{aligned}
P_{0} & =\frac{p_{0}}{R} \sin \beta \\
Q_{0} & =\left(1-P_{0}^{2}\right)^{1 z^{2}}
\end{aligned}
$$

By an evident trigonometric transformation

$$
\begin{aligned}
& \sin \left(\alpha-\alpha_{0}\right)=P Q_{0}-P_{0} Q \\
& \cos \left(\alpha-\alpha_{0}\right)=P P_{0}+Q Q_{0}
\end{aligned}
$$

Substituting the values from equation (8) in equation (2)

$$
\begin{aligned}
& x=\left(P P_{0}+Q Q_{0}\right) q-\left(P Q_{0}-P_{0} Q\right) d \\
& y=\left(P Q_{0}-P_{0} Q\right) q+\left(P P_{0}+Q Q_{0}-1\right) d
\end{aligned}
$$

Equations (9) enable the coordinates of any desired point on the curve to be obtained, but the form remains an inconvenient one for the present purpose. If $x_{1}, y_{1}$ be the coordinates of a given point on the curve, $r$ may be defined by the equation

$$
r=\frac{x_{1}^{2}+y_{1}^{2}}{2 y_{1}}
$$

where $r$ is the radius of curvature of a circle which cuts the axis of the lathe at right angles at the origin and passes through the point $x_{1}, y_{1}$. If the generated curve were truly circular the value of $r$ would be independent of the particular point $x_{1} y_{1}$ selected. As has been mentioned, however, the generated curve is only approximately circular and $r$ is a function of $x_{1} y_{1}$.

Substitution from equations (9) may be made directly in equation (10). However, a more convenient form is obtained by a series development. Two auxiliary quantities are defined by the equations

$$
\begin{aligned}
\rho & =\frac{Q_{0} R}{2 \sin \beta} \\
n & =q / d
\end{aligned}
$$

From equations (1), (3), (7), and (11) one may write

$$
P=P_{0}+\frac{n d Q_{0}}{2 \rho}
$$

and; by substitution in equation (3), $Q$ may be expressed as an infinite series and $P_{0}$ and $Q_{0}$.

By making these substitutions for $P$ and $Q$ in equations (9) and substituting these values of $x$ and $y$ for $x_{1}$ and $y_{1}$ in equation (10), a series development for $r$ may be obtained of which the first few terms are given in equation (13).

$$
\begin{aligned}
r=\rho-\left(\frac{3}{4}+\frac{1}{4} \frac{P_{0}}{Q_{0}} n\right) d+ & \frac{1}{16}\left[1-\left(\frac{P_{0}}{Q_{0}}\right)^{2} n^{2}\right] \frac{d^{2}}{\rho} \\
& -\frac{1}{64}\left(n \frac{P_{0}}{Q_{0}}-1\right)\left[2 \frac{P^{2}}{Q_{0}{ }^{2}} n^{2}+2 \frac{P_{0}}{Q_{0}} n+n^{2}+1\right] \frac{d^{3}}{\rho^{2}}
\end{aligned}
$$

The right-hand member is an implicit function of $\beta$ as will be noted by reference to equations (7) and (11). In the applications which have 
been made this series is so rapidly convergent that it is necessary to retain only the first three terms and $\frac{P_{0}}{Q_{0}}$ changes so slowly for changes in $r$ that the value of $\beta$ corresponding to any value of $r$ may be readily obtained. The angle $\beta$, as indicated in Figure 1, is considered positive, $r$ and $\rho$ of equation (16), for this case, are positive and a convex surface is produced. If the slope of the line $D E$ changes sign, $\beta, r$ and $\rho$ are negative and a concave surface is produced.

\section{DIMENSIONS AND PERFORMANCE CHARACTERISTICS OF LINKAGE ADOPTED FOR CONSTRUCTION}

The instrument which has been built enables tools $300 \mathrm{~mm}$ in diameter to be produced and permits the turning of either convex or concave surfaces with the radius of curvature greater than $500 \mathrm{~mm}$. This range of curvatures is considered entirely satisfactory as more sharply curved surfaces may be readily and conveniently produced by mounting the tool on the end of a radius rod.

The constants which were adopted for the instrument are

$$
\begin{aligned}
\alpha_{0} & =45^{\circ} \\
R & =150 \sqrt{2} \mathrm{~mm} \\
d & =50 \mathrm{~mm}
\end{aligned}
$$

and the maximum value of $n$ is 3 . Reference to Figure 1 indicates that

From equation (7)

$$
p_{0}=150 \cot \beta+150
$$

$$
\begin{aligned}
& P_{0}=\frac{\cos \beta}{\sqrt{2}}+\frac{\sin \beta}{\sqrt{2}}=\sin (45+\beta) \\
& Q_{0}=\quad \cos (45+\beta)
\end{aligned}
$$

and equation (13), with the last term omitted, becomes

$$
r=\rho-\frac{3}{4} d-\frac{1}{4} n d \tan \left(45^{\circ}+\beta\right)+\frac{1}{16} \frac{d^{2}}{\rho}-\frac{1}{16} \frac{n^{2} d^{2}}{\rho} \tan ^{2}\left(45^{\circ}+\beta\right)
$$

As has been already mentioned, the surfaces which are generated by this apparatus are approximately but not strictly spherical. In equation (16) this is evidenced by the presence of $n$ and $n^{2}$ in the right-hand member. In investigating this departure from sphericity several assumptions will be made in order to simplify the work without any important lessening of the accuracy.

1. The last two terms in equation (16) will be neglected.

2. It will be assumed that $x$ of the coordinate system indicated in Figure 1 is equal to $n d$. Reference to the first of equations (2) will indicate the approximation involved. ${ }^{1}$

3. The sagitta of the approximately circular arc, $K L$, shown in Figure 2 will be assumed equal to $\frac{x^{2}}{2 r}$.

4. In the quantity $(4 \rho+3 d)$, which will occur, it will be assumed that $\rho$ can be replaced by $r+60$. 
If $n_{1} d$ is the radius of the tool to be turned, the radius of curvature of the edge zone, as it has been defined, will be approximately yiven by the equation

$$
r_{1}=\rho-\frac{3}{4} d-\frac{1}{4} n_{1} d \tan \left(45^{\circ}+\beta\right)
$$

The sagitta of a sperical surface, having this radius of curvature, at a distance $n d$ from the center, will be

$$
\frac{2 n^{2} d^{2}}{4 \rho-3 d}\left[1+\frac{n_{1} d \tan \left(45^{\circ}+\beta\right)}{4 \rho-3 d}\right]
$$

and, similarly, the sagitta of the actual surface, at the same distance from the center, will be

$$
\frac{2 n^{2} d^{2}}{(4 \rho-3 d)}\left[1+\frac{n d \tan \left(45^{\circ}+\beta\right)}{4 \rho-3 d}\right]
$$

The distance between these two surfaces, which will be/termed the departure from sphericity, for the zone $n d$ will be

$$
\frac{2 n^{2} d^{3}\left(n_{1}-n\right)}{(4 \rho-3 d)^{2}} \tan \left(45^{\circ}+\beta\right)
$$

Applying assumption 4 one may write

Departure from sphericity

$$
\left.=\frac{2\left(n_{1} n^{2}-n^{3}\right) d}{(4 r+90}\right)^{2} \tan \left(45^{\circ}+\beta\right)
$$

For given values of $r$ and $n_{1}$, this expression will assume a maximum value when $n=\frac{2}{3} n_{1}$ and, substituting this value

Maximum departure from sphericity

$$
=\frac{8}{27} \frac{n^{3}{ }_{1}^{3}}{(4 r+90)^{2}} \tan \left(45^{\circ}+\beta\right)
$$

This expression gives the maximum distance, measured parallel to the axis of the tool, between the actual surface as turned and a spherical surface with a radius of curvature corresponding to the zone of radius $n_{1} d$ and is a measure of the thickness of metal which must be removed to obtain a rigorously spherical surface. For a given radius of curvature it is evident that this departure varies as the cube of the diameter of the tool and inversely, approximately as the square of the radius of curvature. Consequently, within the working range of the instrument, the greatest values of the departure from sphericity will be obtained when the diameter of the tool is 300 $\mathrm{mm}\left(n_{1}=3\right)$ and when $r$ equals +500 or $-500 \mathrm{~mm}$. For $r$ equals +500 (convex surface) and $r$ equals -500 (concave surface), $\beta$ assumes the values $+6.7^{\circ}$ and $-11.4^{\circ}$, respectively, and tangent $\left(45^{\circ}+\beta\right)$ assumes the values 1.27 and 0.55 . This indicates that a 
concave surface generated by this attachment will, in general, be more nearly spherical than a convex surface although, as the absolute value of $r$ increases, $\beta$ approaches zero as a limit and the values of tan $\left(45^{\circ}+\beta\right)$ approach equality for the two types of surfaces. For $n_{0}=3$ and $r=+500$ or -500 , the maximum departures from sphericity are

For convex surface $m m$

For concave surface 0.3 15

In considering these values of the departure from sphericity it should be remembered that these are the extreme values for the most unfavorable conditions and that the departures are very much less for the greater part of the working range of the instrument. For example, for tools $200 \mathrm{~mm}$ in diameter and $r$ equals $+1,000$ or $-1,000 \mathrm{~mm}$ the departures from sphericity are

For convex surface. 0.02

For concave surface. 01

In equation (16) for all values of $\beta$ and $\rho$ lying within the range of the instrument, the third term of the right-hand member is the term which determines the sense in which the surface departs from true sphericity. This term does not change sign, and, accordingly, for a convex surface, the absolute value of the radius of curvature decreases from the center outward and for a concave surface the change is in the opposite direction. Consequently the marginal portions of a convex surface and the central portions of a concave surface are the more strongly curved.

In Table 1 , the values of $\beta$ corresponding to different values of $r$ are tabulated. These values are determined for $n=1.8$ and the values of $\beta$, as tabulated, may be used for tools $200 \mathrm{~mm}$ or less in diameter. For convex surfaces approaching $300 \mathrm{~mm}$ in diameter it is well to increase the desired value of $r$ by 15 and to use this new value when entering the table. ${ }^{2}$ Similarly, for a concave surface the absolute value of the desired $r$ should be decreased by 10 before entering the table. For values of $r$ greater than $10,000 \mathrm{~mm}$, the value of $\beta$ can be computed to a satisfactory approximation by the formula

$$
\tan \beta=\frac{75}{r+135}
$$

where $r$ and $\beta$ are positive for convex, negative for concave surfaces.

${ }^{2}$ As has been noted, Table 1 is computed for $n=1.8$. For a tool approaching $300 \mathrm{~mm}$ in diameter it is desirable that the surface actually turned and the desired spherical surface coincide for a zone further from the center than that corresponding to $n=1.8$. For a convex surface, this result will be attained if the value of $r$ for $n=1.8$ is made $15 \mathrm{~mm}$ greater than the nominal value desired for the complete surface. Similarly, for the concave surface, the value of $r$ for $n=1.8$ is less than the value corresponding to the complete surface. 
TABLE 1. - Values of $\beta$ corresponding to $r$

\begin{tabular}{|r|c|c|}
\hline $\boldsymbol{r}$ & $\begin{array}{c}\text { Convex } \\
\text { surface } \\
\boldsymbol{\beta}\end{array}$ & $\begin{array}{c}\text { Concave } \\
\text { surface } \\
\boldsymbol{\beta}\end{array}$ \\
\hline$m m$ & $\circ$ & $\circ$ \\
500 & 6.66 & -11.38 \\
600 & 5.78 & -9.04 \\
700 & 5.10 & -7.49 \\
800 & 4.56 & -6.39 \\
900 & 4.13 & -5.57 \\
1,000 & 3.77 & -4.93 \\
2,000 & 2.01 & -2.30 \\
3,000 & 1.37 & -1.50 \\
4,000 & 1.04 & -1.11 \\
$\mathbf{5}, 000$ & .84 & -.88 \\
6,000 & .70 & -.73 \\
7,000 & .60 & -.63 \\
8,000 & .53 & -.55 \\
9,000 & .47 & -.48 \\
10,000 & .42 & -.44 \\
\hline
\end{tabular}

\section{MECHANICAL DETAILS OF THE COMPLETED INSTRU- MENT}

The attachment has been built in such form that it replaces the usual compound slide rest on the lathe. No other alterations are necessary and the lathe may be used interchangeably for ordinary turning or the facing of spherical surfaces. In Figure 1 the constraints have been represented as pins against which the sides of the triangle bear. In the mechanical realization of this linkage the pins are necessarily replaced by pivoted slides of sufficient strength to oppose the forces on the cutting tool. The detailed design, as completed at the Bureau of Standards, is shown in Figure 2. The tool, indicated at $L$, is carried on a cross slide pivoted at $A$. The pivot at $A$ corresponds to the similarly lettered constraint in Figure 1 and the line $C D$ corresponds to the line $C D$ of the triangle. The member which carries the tool is extended to the right of the slide and carries a sector $F G B$, pivoted at $B$. This sector is rotated about $B$ and may be set in any desired position by means of the worm which is rotated by the handle at $I$. The angular setting of the sector can be read by an arc graduated in degrees and by a graduated drum which is mounted on the shaft of the worm. On the lower surface of the sector there is a male dovetailed member, indicated by the dotted lines parallel to the line DE of Figure 1. This dovetailed member corresponds to the side $D E$ of the triangle of Figure 1 and it can be set in the required position for any desired radius of curvature by rotating the sector. The arc, carried by the sector, is graduated to permit the value of $\beta$ to be read directly. In Figure 2 the setting illustrated corresponds to $\beta=0, C D$ and $D E$ are parallel and the instrument will produce a plane surface. The dovetailed member which has been mentioned slides in a short dovetailed slide pivoted in the base at $B$. The small section in Figure 3 indicates the details of this arrangement. The pivots at $A$ and $B$, corresponding to the constraints $A$ and $B$, respectively, of Figure 1 , are carried by the base of the attachment and remain invariant in position with respect to each other. It will be noted that the pivot about which the sector rotates is directly over the pivot at $B$ when the inain slide is at right 
angles to the axis of the lathe and the point of the tool is on the axis of the lathe. Consequently, when in this position, the sector can be rotated through any desired angle without causing any rotation of the line $D C$ about $A$.

As has been mentioned, this attachment is mounted on the carriage of the lathe in place of the compound slide rest. Reference to a table, such as Table 1, gives the required value of $\beta$ for the desired radjus

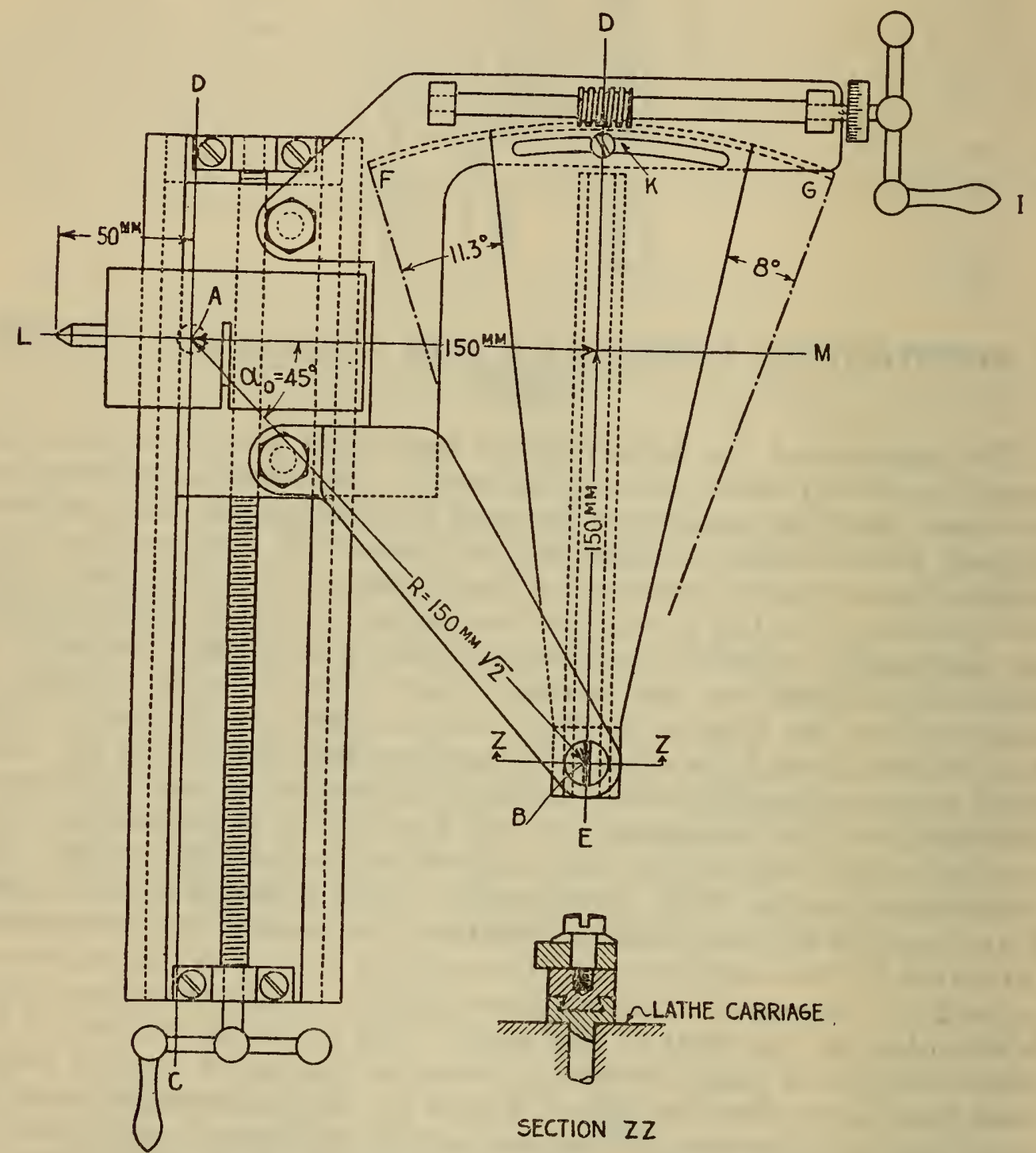

FIGURE 2.-Mechanical realization of linkage

and the setting is made by turning the handle $I$, after which the sector is clamped in position by the nut at $K$. The cut is then made in the uaual manner by turning the handle at $I$. To advance the tool for a second cut, the entire attachment is advanced toward the work by traversing the carriage along the bed of the lathe. The operation of facing a spherical surface with this attachment is quite as simple as facing a plane surface with a compound slide rest except that no automatic cross-feed is provided. This could be easily added 
One of these attachments has been built by the Mann Instrument Company, Cambridge, Miass. Although the details of the design shown in Figure 2 have been modified to correspond to their practice in instrument design, the fundamental dimensions and elements of design were retained unaltered Figures 3 and 4 give two views of the completed attachment mounted on a lathe in position for operation. In the two views the different details of construction to which reference has been made are clearly recognizable. The attachment has proved quite satisfactory for the production of surfaces for lens, tools, and similar purposes.

WASHINGTON, June 2, 1932. 